\title{
Abdominal obesity: a marker of ectopic fat accumulation
}

\author{
Ulf Smith
}

The Lundberg Laboratory for Diabetes Research, Department of Molecular and Clinical Medicine, Sahlgrenska Academy at the University of Gothenburg, Gothenburg, Sweden.

\begin{abstract}
In the early 1980s, we analyzed the metabolic profile of 930 men and women and concluded that an abdominal distribution of fat for a given BMI is associated with increased insulin resistance and risk of developing type 2 diabetes and cardiovascular disease. The correlation between abdominal fat and metabolic dysfunction has since been validated in many studies, and waist circumference is now a criterion for the diagnosis of metabolic syndrome. Several mechanisms for this relationship have been postulated; however, we now know that visceral fat is only one of many ectopic fat depots used when the subcutaneous adipose tissue cannot accommodate excess fat because of its limited expandability.
\end{abstract}

\section{Adipose tissue distribution and metabolic complications of obesity}

As a medical student interested in science, I was fortunate to meet the late Per Björntorp in Sweden, who was at the time an established scientist in the field of obesity research. Along with Per Björntorp, Jules Hirsch and Lester Salans at The Rockefeller University were some of the early and influential investigators who were interested in adipose tissue growth and expansion and how these processes relate to the metabolic complications of obesity. They established techniques to measure adipose cell size from local tissue biopsies and combined these measurements with total body fat to estimate adipose cell numbers in obese and nonobese individuals $(1,2)$. Studies that used these techniques generated much information about adipose tissue expansion in men and women. Nevertheless, access to adipose tissue was limited at that time to the subcutaneous fat, making these estimates less robust. The Hirsch and Salans group and the Björntorp group also provided early evidence that adipose cells from obese individuals were insulin resistant and suggested that obesity- associated insulin resistance could be a consequence of adipose cell expansion (3).

When I became interested in adipose tissue and its metabolism, Per Björntorp and his students had already established the basic techniques and provided me with a fairly easy ride into this novel area of research. As a physician, I had the opportunity to meet patients with different diseases, including common diabetes and lipodystrophic diabetes. One interesting group of patients had the diagnosis of Werner syndrome, which is a rare disorder characterized by premature aging and partial lipodystrophy (4). Individuals with Werner syndrome frequently develop diabetes, and I had the opportunity to phenotype some of these patients and take local biopsies of the subcutaneous adipose tissue. At that time, Mario DiGirolamo, from Emory University, was on a sabbatical in Sweden and was also involved in these studies. Much to our surprise, we found that the adipose cells in the small amount of abdominal adipose tissue in these patients were the largest we had ever seen in our studies of healthy and obese diabetic individuals (5). Lipolysis in these abnormally large adipose cells was markedly elevated, and, in fact, at the time we suggested that "... a regional abdominal adiposity...may promote the metabolic events and alterations that are typically observed in generalized forms of obesity" (5). Little did we know how right this hypothesis would turn out to be, albeit in a different way than we imagined!

The concept that abdominal obesity is a particular characteristic of metabolic risk and diabetes had already been suggested in 1953 by Jean Vague in a French journal (6); however, this work was not recognized by non-francophone scientists until much later. Vague noted that android obesity was more commonly associated with diabetes and metabolic aberrations than the gynoid (female) type of obesity (6). The possibility that obesity type is indicative of metabolic risk later became a more generally recognized concept, due at least in part to the fact that different forms of obesity are fairly easy to recognize visually!

\section{A collaborative effort}

During the early 1980s, Per Björntorp's former trainees collectively characterized and treated many obese individuals. The major drivers of this research were Marcin Krotkiewski and Lars Sjöström. In our joint discussions, we decided to pool all results of the measurements in obese individuals and determine whether those with abdominal obesity exhibited greater differences in metabolic risk profile than individuals with peripheral/lower-body obesity with the same amount of total body fat. This collaborative effort was the beginning of the work that we published in the JCI in 1983 (7). While our work characterizing 930 men and women was under way, the late Ahmed Kissebah and his group in Wisconsin published a paper in 1982 that demonstrated a relationship between obesity type and metabolic complications in a study of 34 women (8). At the time the Kissebah paper was published, we had mostly completed our analyses and tried to rapidly put the data together in the paper 


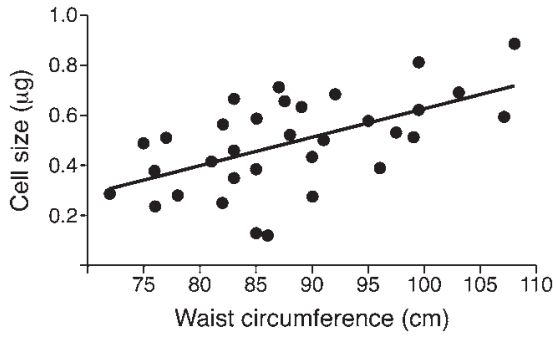

Figure 1. Association between waist circumference and SAT adipose cell size. Waist circumference and SAT adipose cell size were determined in healthy, nonobese $\left(\mathrm{BMI}<30 \mathrm{~kg} / \mathrm{m}^{2}\right)$ men. Data are from ref. 16.

we submitted to the JCI in August of 1982. By analyzing such a large group of obese men and women, we could make a clear case that "men and women with a male abdominal type of obesity are more susceptible to the effect of excess body fat on lipid and carbohydrate metabolism" (7). The concept that individuals with abdominal obesity are more susceptible to metabolic dysfunction has since become textbook knowledge.

In our JCI study, we used waist/hip circumference as a marker of relative abdominal fat distribution, and this measure has since been determined to be a better marker of future cardiovascular disease (CVD) and type 2 diabetes (T2D) risk than BMI. Moreover, J.-P. Després and colleagues in Quebec have expanded on this concept and characterized future risk for CVD and diabetes by measuring waist circumference and relating it to BMI (9). This combinatorial approach revealed that a large waist circumference increases the future risk of CVD and diabetes by two- to three-fold for a given BMI (9).

There has been an extensive effort to try to understand the mechanisms by which regional abdominal fat distribution, irrespective of the total amount of body fat, leads to metabolic complications. One proposed concept (the portal hypothesis) emphasized the importance of visceral/intra-abdominal fat itself, rather than subcutaneous fat, and was based on venous drainage of visceral/intra-abdominal fat into the portal circulation and the finding that high free fatty acid (FFA) levels antagonize the effect of insulin in the liver by reducing receptor-related uptake and signaling (10). Subsequent work has since challenged the portal hypothe- sis and changed views on the metabolic importance of visceral/intra-abdominal fat itself. For instance, the excellent work by Michael Jensen at the Mayo Clinic has shown that compared with subcutaneous fat, the relatively small amount of visceral fat is not a major contributor to overall FFA release or delivery to the liver (11). In addition, no human studies have shown that FFA levels in portal blood are higher than those in the peripheral circulation.

\section{The link to ectopic fat accumulation}

Why, then, does an abdominal adipose tissue distribution mirror metabolic risk? As is frequently the case, the mechanisms that support this relationship are intricate and complex, and abdominal adipose tissue distribution is now considered merely a marker of these underlying mechanisms. Several large prospective studies indicate that subcutaneous adipose tissue is the largest and least metabolically harmful storage site of excess fat. For example, the large prospective Dallas Heart Study found that ectopic fat accumulation, rather than the amount of subcutaneous fat, predicts future development of T2D (12).

Other detailed studies have shown that increased visceral/intra-abdominal fat is a marker of increased ectopic fat in other sites, such as the liver and the heart (13); therefore, abdominal fat distribution can now be considered a marker of ectopic fat in many sites. Moreover, these studies support ectopic fat, and not subcutaneous fat, as the driver of metabolic complications. It remains to be determined whether metabolic complications arise as a result of accumulation of ceramide or other lipids, such as diacyl glycerol, that are associated with mitochondrial dysfunction (14). Subsequent studies have shown that individuals with a preponderance of abdominal fat and a large waist circumference are characterized by both increased visceral/ intra-abdominal fat and expanded (hypertrophic) subcutaneous adipose cells, combined with dysfunctional and inflamed adipose tissue (15). Moreover, there is a direct correlation between waist circumference and subcutaneous adipose cell size (16) (Figure 1). The expansion of the subcutaneous cells is a consequence of the limited ability of this tissue to recruit new adipose cells (17), leading to dysfunc- tional subcutaneous adipose tissue (SAT) and increased ectopic fat accumulation. Importantly, a recent study has shown that genetic predisposition for T2D, which is defined by the Foundation for Diabetes Research as being a first-degree relative of an individual with T2D, is characterized by impaired SAT adipogenesis and increased waist circumference (18). Together, this recent work finally begins to lead us to a comprehensive understanding of why abdominal fat distribution is a marker of future metabolic risk.

\section{Conclusions and future directions}

There is likely a genetic component that determines the ability to accommodate excess fat in the SAT, and if this capacity is exceeded, the resulting compound effects of the expanded SAT are an increase in ectopic intra-abdominal/visceral fat accumulation caused by dysfunctional and hypertrophic SAT and a subsequent increase in waist circumference. Future work should focus on understanding genetic and other factors that regulate SAT adipogenesis and how subcutaneous adipocyte generation could be improved to accommodate excess fat and prevent ectopic fat accumulation. Interestingly, known genetic risk factors for insulin resistance and T2D have been identified in individuals with a lower body weight but characterized by increased markers of ectopic fat accumulation (19). The prevention of ectopic fat accumulation and its diverse consequences, including nonalcoholic fatty liver disease (NAFLD), nonalcoholic steatohepatitis (NASH), cirrhosis of the liver, T2D, and CVD, is a major challenge, but one of great importance for public health.

\section{Acknowledgments}

We wish to thank the Swedish Research Council, the Swedish Diabetes Association, the Novo Nordisk Foundation, the Torsten Söderberg Foundation, and the West Sweden ALF program for financial support.

Address correspondence to: Ulf Smith, Department of Molecular and Clinical Medicine, Sahlgrenska University Hospital, Vita Straket 12, SE-413 45 Gothenburg, Sweden. Phone: 46.31.3421104; E-mail: ulf.smith@medic.gu.se. 
1. Björntorp P, Gustafson A, Persson B. Adipose tissue fat cell size and number in relation to metabolism in endogenous hypertriglyceridemia. Acta Med Scand. 1971;190(5):363-367.

2. Hirsch J, Knittle JL. Cellularity of obese and nonobese human adipose tissue. Fed Proc. 1970;29(4):1516-1521.

3. Salans LB, Knittle JL, Hirsch J. The role of adipose cell size and adipose tissue insulin sensitivity in the carbohydrate intolerance of human obesity. JClin Invest. 1968;47(1):153-165.

4. Oshima J, Hisama FM. Search and insights into novel genetic alterations leading to classical and atypical Werner syndrome. Gerontology. 2014;60(3):239-246.

5. Smith U, Digirolamo M, Blohme G, Kral JG, Tisell LE. Possible systemic metabolic effects of regional adiposity in a patient with Werner's syndrome. Int JObes. 1980;4(2):153-163.

6 . Vague J. [Significance of obesity in medical practice]. Mars Med.1953;90(4):179-189.

7. Krotkiewski M, Björntorp P, Sjöström L, Smith U.
Impact of obesity on metabolism in men and women. Importance of regional adipose tissue distribution. J Clin Invest. 1983;72(3):1150-1162.

8. Kissebah AH, et al. Relation of body fat distribution to metabolic complications of obesity. JClin Endocrinol Metab. 1982;54(2):254-260.

9. Després JP, Lemieux I. Abdominal obesity and metabolic syndrome. Nature. 2006;444(7121):881-887.

10. Svedberg J, Strömblad G, Wirth A, Smith U, Björntorp P. Fatty acids in the portal vein of the rat regulate hepatic insulin clearance. J Clin Invest. 1991;88(6):2054-2058.

11. Jensen MD, Cardin S, Edgerton D, Cherrington A. Splanchnic free fatty acid kinetics. Am J Physiol Endocrinol Metab. 2003;284(6):E1140-E1148.

12. Neeland IJ, et al. Dysfunctional adiposity and the risk of prediabetes and type 2 diabetes in obese adults. JAMA. 2012;308(11):1150-1159.

13. Graner M, et al. Cardiac steatosis associates with visceral obesity in nondiabetic obese men. J Clin Endocrinol Metab. 2013;98(3):1189-1197.
14. Szendroedi J, et al. Role of diacylglycerol activation of PKCtheta in lipid-induced muscle insulin resistance in humans. Proc Natl Acad Sci US A. 2014;111(26):9597-9602.

15. Klöting N, et al. Insulin-sensitive obesity. Am J Physiol Endocrinol Metab. 2010;299(3):E506-E515.

16. Hammarstedt A, et al. WISP2 regulates preadipocyte commitment and PPARgamma activation by BMP4. Proc Natl Acad Sci US A. 2013;110(7):2563-2568.

17. Gustafson B, Smith U. The WNT inhibitor Dickkopf 1 and bone morphogenetic protein 4 rescue adipogenesis in hypertrophic obesity in humans. Diabetes. 2012;61(5):1217-1224.

18. Arner P, Arner E, Hammarstedt A, Smith U. Genetic predisposition for type 2 diabetes, but not for overweight/obesity, is associated with a restricted adipogenesis. PLoS One. 2011;6(4):e18284.

19. Scott RA, et al. Common genetic variants highlight the role of insulin resistance and body fat distribution in type 2 diabetes, independent of obesity. Diabetes. 2014;63(12):4378-4387. 